\title{
TITLE:
}

\section{Theoretical study on the ionization of aniline in aqueous solutions}

$\operatorname{AUTHOR}(S)$ :

Matsumura, Yoshihiro; lida, Kenji; Sato, Hirofumi

\section{CITATION:}

Matsumura, Yoshihiro ...[et al]. Theoretical study on the ionization of aniline in aqueous solutions. Chemical Physics Letters 2013, 584: 103107

ISSUE DATE:

2013-10-01

URL:

http://hdl.handle.net/2433/179298

\section{RIGHT:}

@ 2013 Elsevier Ltd.; この論文は出版社版でありません。引用の際には 出版社版をご確認ご利用ください。; This is not the published version. Please cite only the published version. 


\title{
Theoretical study on the ionization of aniline in aqueous solutions
}

\author{
Yoshihiro Matsumura ${ }^{\mathrm{a}}$, Kenji Iida ${ }^{\mathrm{a}, 1}$, Hirofumi Sato ${ }^{\mathrm{a}, \mathrm{b}, *}$ \\ ${ }^{a}$ Department of Molecular Engineering, Graduate School of Engineering, Kyoto University, Nishikyo-ku, Kyoto 615-8510, \\ Japan \\ ${ }^{b}$ Elements Strategy Initiative for Catalysts and Batteries (ESICB), Kyoto University, Nishikyo-ku, Kyoto 615-8510, Japan
}

\begin{abstract}
The ionization and excitation processes of aniline in aqueous solutions are investigated by the method of RISM-SCF-SEDD(reference interaction site model self-consistent field with the spatial electron density distribution). Four different models are employed to characterize the response of the solvation upon the ionization and excitation. A simple treatment for estimation of the spectral width is also proposed.
\end{abstract}

\section{Introduction}

Ionization potential (IP) is one of the fundamental quantities to characterize the electronic structures of molecules. As precise IP values have recently become available for molecules in the solution phase [13], significant shifts have been observed for molecules in aqueous solutions from those in the gas phase. These results strongly indicate that the electronic structures of the molecules are significantly influenced by solvation.

The effects of solvation on ionization have been studied theoretically by means of the electronic structure theory combined with a variety of solvation theories [2-9]. The IPs of DNA and RNA bases were calculated in aqueous solutions using the polarizable continuum model (PCM) $[2,3,9,10]$. However, the role of local interaction such as hydrogen bonding is not sufficiently understood due to the simplification of the model. The IP and the spectral width of aqueous phenol were calculated using the quantum mechanics/molecular mechanics (QM/MM) simulation combined with the dielectric continuum treatment [11], but this theory requires a high cost of computation for the generation of the solvent configurations in thermodynamical equilibrium.

We wish to report here the effects of solvation on the electronic transition of aniline with particular focus on ionization. Aniline is the simplest aromatic amine, and its electronic structure has been studied in detail both experimentally and theoretically [12-15]. However, little is yet known on the interactions in solvated aniline despite the vital importance from the viewpoint of a biologically relevant model system.

\footnotetext{
${ }^{*}$ Corresponding author. Tel: +81 75 3832548. Fax: +81 753832799.

Email address: hirofumi@moleng.kyoto-u.ac.jp (Hirofumi Sato)

${ }^{1}$ Present address: Institute for Molecular Science, Okazaki, 444-8585, Japan 
The RISM-SCF-SEDD method [16-19] has been applied in the present Letter to investigate the solvation effects on the IP values of aniline. The reference interaction site model self-consistent field (RISM-SCF) is the method that combines the statistical mechanics of molecular liquids (RISM) [20] and ab initio molecular orbital theory. Thanks to the analytical nature of RISM, the solvation structure can be computed in the thermodynamical equilibrium state with a reasonable computational cost. The RISM-SCF method has achieved clarification of the solvation effect on molecular electronic structures [4-8, 21-25]; the ionization processes of dimethyl aniline $[7,8]$ and the excitation of $p$-nitro aniline in water over a wide range of density condition [24] are examples. We have used RISM-SCF in the present study with the method of spatial electron density distribution (RISM-SCF-SEDD) $[18,19]$; this is an extended method of the original RISMSCF by explicit inclusion of electron density distribution. In contrast to the original RISM-SCF, RISMSCF-SEDD method is numerically stable in the convergence, even if the buried atoms are involved. Please refer Ref[18] for more details. The method has been applied to a wide range of chemical processes. To our knowledge, this is the first theoretical study of the solvation effects on aniline.

\section{Method}

\subsection{RISM-SCF-SEDD method}

Let us first summarize the RISM-SCF-SEDD method, which combines the statistical mechanics of molecular liquids (RISM) with the ab initio molecular orbital theory. The equation of RISM is given as

$$
\mathbf{h}^{\mathrm{UV}}=\boldsymbol{\omega}^{\mathrm{U}} * \mathbf{c}^{\mathrm{UV}} * \boldsymbol{\omega}^{\mathrm{V}}+\omega^{\mathrm{U}} * \mathbf{c}^{\mathrm{UV}} * \boldsymbol{\rho}^{\mathrm{V}} \mathbf{h}^{\mathrm{VV}}
$$

Here ' $*$ ' denotes convolution integral. Symbols $\boldsymbol{\omega}^{\mathrm{U}}$ and $\boldsymbol{\omega}^{\mathrm{V}}$ are intramolecular correlation functions of solute and solvent, $\mathbf{h}^{\mathrm{UV}}$ and $\mathbf{c}^{\mathrm{UV}}$ are the total and direct correlation functions between solute and solvent, respectively, and $\mathbf{h}^{\mathrm{VV}}$ is the total correlation function for solvent with the number density $\boldsymbol{\rho}^{\mathrm{V}}$. Hyper-netted chain (HNC) closure is adopted in the present study to solve the equation,

$$
h_{\alpha s}^{\mathrm{UV}}=\exp \left(-\beta u_{\alpha s}^{\mathrm{UV}}+h_{\alpha s}^{\mathrm{UV}}-c_{\alpha s}^{\mathrm{UV}}\right)-1
$$

where $\beta=1 / k_{B} T, k_{B}$ is the Boltzmann constant and $u_{\alpha s}^{U V}$ is the interaction between site $\alpha$ and $s$. Under the HNC approximation, solvation free energy $(\Delta \mu)$ is expressed as

$$
\Delta \mu=\beta^{-1} \rho^{\mathrm{V}} \sum_{\alpha, s} \int_{0}^{\infty} 4 \pi r^{2} \mathrm{~d} r\left[\frac{1}{2}\left(h_{\alpha s}^{\mathrm{UV}}\right)^{2}-\frac{1}{2} c_{\alpha s}^{\mathrm{UV}} h_{\alpha s}^{\mathrm{UV}}-c_{\alpha s}^{\mathrm{UV}}\right] .
$$

In RISM-SCF-SEDD, the solvated Fock operator is defined by

$$
\widehat{F}=\widehat{F}^{\text {gas }}-\widehat{V}
$$

where $F^{\text {gas }}$ is the Fock operator of an isolated molecule, $V$ is the solute-solvent electrostatic interaction operator. The electrostatic interaction energy between the solute and solvent is written as

$$
\langle\Psi|\widehat{V}| \Psi\rangle=\sum_{j} D_{j} V_{j}=\mathbf{D} \cdot \mathbf{V},
$$


and $\Psi$ is the wave function of the solute molecule in the solution phase. The expansion coefficients, $D_{j}$, of the charge density correspond to the net population of electrons in the $j$-th auxiliary basis set $\left(\mathrm{ABS}, f_{j}\right)$, and $V_{j}$ is the electrostatic potential on this $\operatorname{ABS}\left(f_{j}\right)$ from the solvent,

$$
\widehat{V}_{j}=\rho^{\mathrm{V}} \sum_{s} q_{s} \int \frac{f_{j}(r)}{r} h_{\alpha s}^{\mathrm{UV}}(r) 4 \pi r^{2} \mathrm{~d} r . \quad(j \in \alpha)
$$

\subsection{Electronic transition energy}

The electronic transition energy in the gas $\left(\Delta E^{\text {gas }}\right)$ is given as the difference in the total energy between the initial $\left(E^{\mathrm{i}}\right)$ and final $\left(E^{\mathrm{f}}\right)$ states,

$$
\Delta E_{X}^{\mathrm{gas}}=E_{X}^{\mathrm{f}}-E^{\mathrm{i}} . \quad(X=\mathrm{I}, \mathrm{II}, \mathrm{III} \text { and IV })
$$

The transition energy in aqueous solution is expressed as a sum of Eq. (7) and the contribution from the solute-solvent interaction, but the former is changed due to the electronic distortion caused by the solvation. Based on Ref. [5], the following four approximated formulae are considered in the present study to investigate the contributions of electronic and geometric relaxations in the ionization process.

As the first model stage, the solvent and the geometry of solute molecule are assumed to be completely frozen at the initial equilibrated state upon the transition. While the electronic structure of the solute molecule is changed on the transition, the polarization of solvent is not allowed. Namely, the electrostatic field generated by solvent is fixed at the initial one, $\mathbf{V}^{\mathrm{i}}$.

$$
\Delta E_{\mathrm{I}}^{\mathrm{aq}}=\Delta E_{\mathrm{I}}^{\mathrm{gas}}+\left(\mathbf{D}_{\mathrm{I}}^{\mathrm{f}}-\mathbf{D}^{\mathrm{i}}\right) \cdot \mathbf{V}^{\mathrm{i}}
$$

This treatment is named "model I". The second stage takes into account a part of the solvent polarization in an effective manner (model II).

$$
\Delta E_{\mathrm{II}}^{\mathrm{aq}}=\Delta E_{\mathrm{II}}^{\mathrm{gas}}+k_{\text {slow }}\left(\mathbf{D}_{\mathrm{II}}^{\mathrm{f}}-\mathbf{D}^{\mathrm{i}}\right) \cdot \mathbf{V}^{\mathrm{i}}+\frac{1}{2} k_{\text {fast }}\left(\mathbf{D}_{\mathrm{II}}^{\mathrm{f}} \cdot \mathbf{V}_{\mathrm{II}}^{\mathrm{f}}-\mathbf{D}^{\mathrm{i}} \cdot \mathbf{V}^{\mathrm{i}}\right),
$$

where $\mathbf{V}_{\mathrm{II}}^{\mathrm{f}}$ is the electrostatic field from the solvent equilibrated to the final state. The second and third terms on the right-hand side correspond to slow and fast components of the solvent response, respectively. Symbols $k_{\text {slow }}$ and $k_{\text {fast }}$ are the constants $(0.4426,0.5574)$ calculated from the dielectric permittivity and the optical dielectric permittivity of water solvent. See Ref. [5] for more detailed discussion.

The third treatment allows the solvent to completely relax,

$$
\Delta E_{\mathrm{III}}^{\mathrm{aq}}=\Delta E_{\mathrm{III}}^{\mathrm{gas}}+\left(\Delta \mu_{\mathrm{III}}^{\mathrm{f}}-\Delta \mu^{\mathrm{i}}\right)
$$

The second term on the right-hand side is simply a difference in the solvation free energy between the two equilibrium states under the assumption that the solute molecular geometry remains unchanged (model III). 
Finally, the geometry relaxation of solute molecule is taken into account (model IV). $\Delta E^{\text {gas }}$ is replaced by $\Delta E_{\mathrm{rlx}}^{\mathrm{gas}}$, which is the energy difference between the two optimized geometries of the initial and final states.

$$
\Delta E_{\mathrm{IV}}^{\mathrm{aq}}=\Delta E_{\mathrm{rlx}}^{\mathrm{gas}}+\left(\Delta \mu_{\mathrm{IV}}^{\mathrm{f}}-\Delta \mu^{\mathrm{i}}\right)
$$

This quantity is the adiabatic ionization potential corresponding to the difference in free energy. Namely, the initial and final states are both in their thermodynamic equilibrium states. Note that $\Delta \mu_{\mathrm{IV}}^{\mathrm{f}}$ is calculated at the optimized geometry of the final state, while $\Delta \mu_{\mathrm{III}}^{\mathrm{f}}$ and $\Delta \mu^{\mathrm{i}}$ are calculated at the optimized geometry of the initial state.

\section{Computational details}

Geometry optimization and energy calculation were performed using the DFT (B3LYP functional) method with the cc-pVDZ basis set both in the gas and aqueous solution phases, respectively. For calculation of the cation, the unrestricted version with the same basis set was adopted. The low-lying excited states were also computed using MRMP2 with the same basis set at the CASSCF-optimized geometry of the ground state. The active spaces comprised a full set of the valence $\pi$-orbitals and the lone pair of $\mathrm{N}$ atom; eight electrons were distributed over seven orbitals for the neutral species.

Solvation effect was taken into account using the RISM-SCF-SEDD method coupled with HNC closure. Standard Lennard-Jones (LJ) parameters taken from the literature were employed (Table 1). All calculations were performed at $298.15 \mathrm{~K}$ and the density of $1.0 \mathrm{~g} / \mathrm{cm}^{3}$. We utilized the GAMESS program package [26] modified by us to perform the RISM-SCF-SEDD calculation. PCM (polarizable continuum model) was also employed to calculate the ionization potential in aqueous solution, using Gaussian 03. Non-equilibrium PCM was employed for the calculations of models I and II.

\section{Results and discussion}

\subsection{Ionization potential and excitation energy}

Table 2 summarizes the calculated ionization potential and the excitation energies in the gas phase. The ionization potential (IP) with (U)B3LYP/cc-pVDZ calculation, $7.59 \mathrm{eV}$, agrees reasonably with the experimental value $7.72 \mathrm{eV}$ [12], as well as with the SAC-CI value, $7.66 \mathrm{eV}$ [14]. The computed excitation energies also agree with the reported values. This table lists the transition energies to the two low-lying excited states of the neutral aniline $\left(\mathrm{S}_{1}\right.$ and $\left.\mathrm{S}_{2}\right)$ and those of the ionized species $\left(\mathrm{D}_{1}\right.$ and $\left.\mathrm{D}_{2}\right)$.

The $\mathrm{S}_{1}$ and $\mathrm{S}_{2}$ states are both characterized by the $\pi \rightarrow \pi^{*}$ transition. The former is described as the excitation from HOMO $\left(a^{\prime}\right)$ to LUMO $\left(a^{\prime \prime}\right)$ in the Kohn-Sham orbitals of DFT. The latter transition is attributed to that from HOMO $\left(a^{\prime}\right)$ to the next LUMO $\left(a^{\prime}\right.$, usually abbreviated as LUMO+1). The electronic structures of $\mathrm{D}_{1}$ and $\mathrm{D}_{2}$ are also described in terms of one-electron picture. $\mathrm{D}_{1}$ corresponds to the removal of an electron from the next HOMO ( $a^{\prime \prime}$, abbreviated as HOMO-1) of the neutral aniline. In a 
similar manner, $\mathrm{D}_{2}$ is characterized as the ionization from the HOMO-2. The nature of these transitions are the same as those reported by the previous studies including SAC-CI [14] and experiments [13], indicating that the validity of the present computation for the electronic structure.

The results in aqueous solutions are listed in Table 3. The ionization energy computed with models I, II, III and IV are 8.66, 7.40, 6.36 and $5.98 \mathrm{eV}$, respectively, using the DFT method. The MRMP2 method provides essentially the same results. Model I gives higher energy than that in the gas phase by $1.07 \mathrm{eV}$. In this treatment, the ground state is more stabilized by solvation than the ionized state because the solvent equilibrates only the ground state and is not allowed to respond to the ionized state. In fact, the ionization energy is led to $0.19 \mathrm{eV}$ lower than that in the gas phase when the fast electric polarization of the solvent is considered (model II). The ionized state is stabilized by $1.04 \mathrm{eV}$ due to the solvent reorganization. As a result, model III is $1.23 \mathrm{eV}$ lower than that in the gas phase. Furthermore, the ionization potential of model IV is lowered by $0.38 \mathrm{eV}$ from that of model III when the geometry relaxation of solute molecule is allowed. The difference in the components of the solvation free energy $\left(\Delta \mu^{\mathrm{f}}-\Delta \mu^{\mathrm{i}}\right)$ between models III and IV is $0.02 \mathrm{eV}$, which is very small.

The corresponding PCM computations of models I, II, III and IV are respectively 7.52, 6.56, 5.29 and $4.96 \mathrm{eV}$ using the DFT method. All of them show $\sim 1 \mathrm{eV}$ lower than those of RISM-SCF-SEDD at each model. This is fully consistent with Ref. [5], implying that the importance of the stabilization of neutral aniline due to the local interactions such as hydrogen bonding as we shall discuss later.

As for the excitation energies, the solvation effect is more enhanced in the ionized excited states, $D_{1}$, $\mathrm{D}_{2}$, compared to the neutral excited states $\left(\mathrm{S}_{1}\right.$ and $\left.\mathrm{S}_{2}\right)$. This may be readily understood in terms of the difference in the total charge of the solute molecule. The electrostatic filed generated by a charged molecule is generally stronger than that by a neutral one.

Finally, the full width at half maximum of the vertical ionization is computed based on linear response theory $[5,27]$;

$$
\begin{aligned}
\sigma_{\mathrm{I}} & =2 \sqrt{2 \ln 2} \sqrt{\frac{1}{\beta}\left|\Delta E_{\mathrm{I}}^{\mathrm{aq},+\leftarrow 0}+\Delta E_{\mathrm{I}}^{\mathrm{aq},+\rightarrow 0}\right|}, \\
\sigma_{\mathrm{II}} & =2 \sqrt{2 \ln 2} \sqrt{\frac{1}{\beta}\left|\Delta E_{\mathrm{II}}^{\mathrm{aq},+\leftarrow 0}+\Delta E_{\mathrm{II}}^{\mathrm{aq},+\rightarrow 0}\right|} .
\end{aligned}
$$

Here, $\Delta E_{X}^{\mathrm{aq},+\leftarrow 0}$ and $\Delta E_{X}^{\mathrm{aq},+\rightarrow 0}(X=\mathrm{I}$ or II $)$ are the electric transition energy from the neutral to the cation, respectively, and vice versa. The above equations of $\sigma_{\mathrm{I}}$ and $\sigma_{\mathrm{II}}$ are derived from models I and II, respectively. The obtained values are 0.80 and $0.58 \mathrm{eV}$ from the B3LYP computation, and 0.82 and $0.62 \mathrm{eV}$ from MRMP2.

These values are in the same order of magnitude as the reported values for aqueous phenol and phenolate [11]. Similar to the previous study on simple molecular systems [5], the contribution from the fast component makes the spectra width narrower. However, a preliminary computation suggests that the width is broadened 
when the relaxation of solute molecular geometry is taken into account. A further sophisticated study is required for a quantitative discussion on the spectral width of ionization.

\subsection{Solvation effect on the electronic structure}

As shown in Sec. 4.1, the ionization energy is changed by the solvation. Based on Koopmans' theorem, the orbital energy of HOMO is equivalent to the reversed sign of ionization potential. In fact, $1.05 \mathrm{eV}$ stabilization of the HOMO energy $(-5.45 \mathrm{eV} \rightarrow-6.50 \mathrm{eV})$ observed in the (U)B3LYP/cc-pVDZ calculation agrees excellently with the directly computed value, $1.07 \mathrm{eV}$ (model I).

In Table 4 , the Mulliken charges of aniline $\left(\mathrm{S}_{0}\right)$ and the cation $\left(\mathrm{D}_{0}\right)$ are listed. For the isolated molecule in the gas phase, the effective charges of all the atoms are increased upon the ionization. As expected, the most significant change is found in the $\mathrm{N}_{7}$ atom because the electron is removed from the HOMO, which is composed of the $\pi$-orbital of the benzene ring and lone pair orbital of this nitrogen atom (Figure 1 ). In aqueous solution, the electronic structure of aniline is polarized, and the dipole moment is increased from 1.76 to $2.70 \mathrm{D}$. In particular, the negative charge on the nitrogen is more enhanced. Upon the ionization, the change in the effective charges can also be decomposed into four steps: models I, II, III and IV. As the solvation is relaxed to the final state, the electrons assigned to the nitrogen and some carbon atoms are shifted to hydrogen atoms. It is notable that the charges on $\mathrm{C}_{2,3}$ and $\mathrm{N}_{7}$ are relatively sensitive to the change in the solvation, whereas those on $\mathrm{C}_{1}$ and $\mathrm{C}_{4,5}$ arrive at the equilibrium state at an early stage of the relaxation.

\subsection{Solvation structure}

Figure 2(a) displays the radial distribution functions (RDFs) of solvent water hydrogen atom $\left(\mathrm{H}_{\mathrm{w}}\right)$ in the vicinity of the terminus carbon $\left(\mathrm{C}_{6}\right)$. In the neutral species, a small shoulder is found at $2 \AA$, suggesting a weak interaction between the solvent water and this carbon. However, this peak disappears on the ionization. This is because the charge on the $\pi$ orbital of the benzene ring is reduced by the ionization (Table 4), and the electrostatic attraction between the ring and the $\mathrm{H}_{\mathrm{w}}$ atom is then diminished. Figure 2(b) displays RDFs between the aniline nitrogen $\mathrm{N}_{7}$ and $\mathrm{H}_{\mathrm{w}}$. Before the ionization, the peak of a typical hydrogen bonding is found at $2 \AA$, and it disappears upon the ionization. As discussed in the preceding subsection, the charge on $\mathrm{N}_{7}$ is reduced, especially in its lone-pair orbital, and the electrostatic attraction between $\mathrm{N}_{7}$ and $\mathrm{H}_{\mathrm{w}}$ is largely diminished. This is consistent with the results shown above; i.e., it is likely that these two sites are most strongly influenced by the solvation. Being different from the transition energies, the change in the solvation structure caused by the geometrical relaxation of aniline is negligible, and the two RDFs corresponding to model III and IV are virtually identical. 


\section{Conclusion}

We have studied the ionization energy of aniline in aqueous solution using the RISM-SCF-SEDD method. The ionization energy outweighs the gas-phase value when the solvent is completely frozen at the equilibrium state of the neutral aniline, but is lowered with the full relaxation of the solvent. The excitation energies to the low-lying states of the neutral molecule are not significantly affected by the solvation. These findings suggest that the ionization energy may be sensitive to the time scale of the electron detachment and observation, whereas the excitation energy is essentially unchanged by the solvation.

The spectral width has also been studied based on the linear response regime proposed recently. The relaxation of solvent seems to make the width narrower, but the reorganization of solute molecules contributes in opposite manner. Although further sophistication is necessary to correctly estimate the width, the present method provides a simple, analytical way to predict the electronic spectra of solvated molecules.

\section{Acknowledgments}

We thank Professor Toshinori Suzuki (Kyoto University) for invaluable discussions. The work was financially supported in part by three Grants-in-Aid for Scientific Research: on Priority Areas "Molecular Science for Supra Functional Systems" (477-22018016), on Innovative Areas "Molecular Science of Fluctuations" (2006-21107511, 23107714), and Research (C) (20550013 and 25410011). The Strategic Programs for Innovative Research (SPIRE), the Computational Materials Science Initiative (CMSI) is also acknowledged. A part of this work was performed under a management of 'Elements Strategy Initiative for Catalysts \& Batteries (ESICB)'. All of them were supported by the Ministry of Education, Culture, Sports, Science and Technology (MEXT) Japan.

[1] B. Winter, M. Faubel, Chem. Rev. 106 (2006) 1176.

[2] B. Jagoda-Cwiklik, P. Slavicak, D. Nolting, B. Winter, P. Jungwirth, J. Phys. Chem. B Lett. 112 (2008) 7355.

[3] P. Slavicak, B. Winter, M. Faubel, S.E. Bradforth, P. Jungwirth, J. Am. Chem. Soc. 131 (2009) 6460.

[4] K. Iida, D. Yokogawa, H. Sato, S. Sakaki, J. Chem. Phys. 130 (2009) 044107.

[5] K. Iida, H. Sato, J. Chem. Phys. 136 (2012) 144510

[6] H. Sato, F. Hirata, J. Phys. Chem. A 106 (2002) 2300

[7] H. Sato, Y. Kobori, S. Tero-Kubota, F. Hirata, J. Chem. Phys. 119 (2003) 2753.

[8] H. Sato, Y. Kobori, S. Tero-Kubota, F. Hirata, J. Phys. Chem. B 108 (2004) 11709

[9] C.E. Crespo-Hernandez, R. Arce, Y. Ishikawa, L. Gorb, J. Leszczynski, D.M. Close, J. Phys. Chem. A 108 (2004) 6373.

[10] J. Tomasi, B. Mennucci, R. Cammi, Chem. Rev. 105 (2005) 2999.

[11] D. Ghosh, A. Roy, R. Seidel, B. Winter, S. Bradforth, A.I. Krylov, J. Phys. Chem. B 116 (2012) 7269.

[12] X. Song, M. Yang, E.R. Davidson, J.P. Reilly, J. Chem. Phys. 99 (1993) 3226.

[13] R. Montero, A.P. Conde, V. Ovejas, R. Martinez, F. Castano, A. Longarte, J. Chem. Phys. 135 (2011) 054308

[14] Y. Honda, M. Hada, M. Ehara, H. Nakatsuji, J. Chem. Phys. 117 (2002) 2045.

[15] P.M. Wojciechowski, W. Zierkiewicz, D. Michalska, P. Hobza, J. Chem. Phys. 118 (2003) 10900.

[16] S. Ten-no, F. Hirata, S. Kato, J. Chem. Phys. 100 (1994) 7443.

[17] H. Sato, F. Hirata, S. Kato, J. Chem. Phys. 105 (1996) 1546.

[18] D. Yokogawa, H. Sato, S. Sakaki, J. Chem. Phys. 126 (2007) 244504.

[19] D. Yokogawa, H. Sato, S. Sakaki, J. Chem. Phys. 131 (2009) 214504.

[20] F. Hirata (Ed.), Molecular Theory of Solvation, Understanding Chemical Reactivity, Springer, 2003.

[21] K. Iida, D. Yokogawa, H. Sato, S. Sakaki, Chem. Phys. Lett. 443 (2007) 264.

$[22]$ K. Iida, D. Yokogawa, A. Ikeda, H. Sato, Phys. Chem. Chem. Phys. 11 (2009) 8556

[23] K. Iida, H. Sato, J. Phys. Chem. B 116 (2012) 2244. 
[24] D. Yokogawa, H. Sato, S. Sakaki, Y. Kimura, J. Phys. Chem. B 114 (2010) 910.

[25] D. Yokogawa, K. Ono, H. Sato, S. Sakaki, Dalton Trans. 40 (2011) 11125.

[26] M.W. Schmidt, K.K. Baldridge, J.A. Boatz, S.T. Elbert, M.S. Gordon, J.H. Jensen, S. Koseki, N. Matsunaga, K.A. Nguyen, S.J. Su, T.L. Windus, M. Dupuis, J.A. Montgomery, J. Comput. Chem. 14 (1993)1347.

[27] J. Åqvist, T. Hansson, J. Chem. Phys. 100 (1996) 9512. 
Table 1: Lennard-Jones parameters of each atom [24].

\begin{tabular}{lrr}
\hline site & $\sigma / \AA$ & $\epsilon / \mathrm{kcal} \mathrm{mol}^{-1}$ \\
\hline solute (aniline) & & \\
$\mathrm{C}$ & 3.550 & 0.070 \\
$\mathrm{H}$ & 2.420 & 0.030 \\
$\mathrm{~N}$ & 3.250 & 0.170 \\
solvent (water) & & \\
$\mathrm{H}$ & 1.000 & 0.056 \\
$\mathrm{O}$ & 3.166 & 0.155 \\
\hline
\end{tabular}

Table 2: Ionization potential $\left(\Delta E^{\text {gas }}\right)$ and excitation energies in the gas phase $(\mathrm{eV})$.

\begin{tabular}{llllll}
\hline & \multirow{2}{*}{ Ionization energy } & \multicolumn{3}{c}{ Excitation energy } \\
\cline { 3 - 5 } & & \multicolumn{5}{c}{$\mathrm{S}_{2}$} & $\mathrm{D}_{1}$ & $\mathrm{D}_{2}$ \\
\cline { 3 - 5 } & 7.59 & - & - & - & - \\
B3LYP & 7.66 & 4.42 & 5.69 & 8.88 & 10.23 \\
MRMP2 & 4.20 & 5.39 & 8.99 & 10.80 \\
SAC-CI [14] & 7.66 & $4.40[13]$ & $5.39[13]$ & - & - \\
Explt. & $7.72[12]$ & &
\end{tabular}

Table 3: Ionization and excitation energies in aqueous solution (eV).

\begin{tabular}{llllllll}
\hline & \multicolumn{2}{c}{ Ionization energy } & & \multicolumn{4}{c}{ Excitation energy } \\
\cline { 2 - 3 } & B3LYP & MRMP2 & & $\mathrm{S}_{1}$ & $\mathrm{~S}_{2}$ & $\mathrm{D}_{1}$ & $\mathrm{D}_{2}$ \\
\hline I & 8.66 & 8.89 & & 4.52 & 5.85 & 9.94 & 11.42 \\
II & 7.40 & 7.73 & & 4.51 & 5.80 & 8.81 & 10.28 \\
III & 6.36 & 6.43 & & 4.48 & 5.66 & 7.63 & 9.05 \\
IV & 5.98 & 5.94 & & & & & \\
\hline
\end{tabular}


Table 4: Gross Mulliken charges of aniline and its cation.

\begin{tabular}{|c|c|c|c|c|c|c|c|c|c|}
\hline & $\mathrm{C}_{1}$ & $\mathrm{C}_{2,3}$ & $\mathrm{C}_{4,5}$ & $\mathrm{C}_{6}$ & $\mathrm{~N}_{7}$ & $\mathrm{H}_{8,9}$ & $\mathrm{H}_{10,11}$ & $\mathrm{H}_{12,13}$ & $\mathrm{H}_{14}$ \\
\hline \multicolumn{10}{|c|}{ in the gas phase } \\
\hline $\mathrm{S}_{0}$ & 0.00 & 0.03 & 0.05 & 0.02 & -0.13 & 0.08 & -0.05 & -0.04 & -0.04 \\
\hline $\mathrm{D}_{0}$ & 0.03 & 0.10 & 0.07 & 0.11 & 0.00 & 0.15 & 0.03 & 0.04 & 0.05 \\
\hline \multicolumn{10}{|c|}{ in aqueous solution } \\
\hline $\mathrm{S}_{0}$ & 0.01 & -0.01 & 0.03 & -0.02 & -0.20 & 0.12 & -0.02 & -0.01 & -0.02 \\
\hline $\mathrm{D}_{0}$ & & & & & & & & & \\
\hline I & 0.03 & 0.07 & 0.06 & 0.07 & -0.06 & 0.19 & 0.06 & 0.07 & 0.07 \\
\hline II & 0.03 & 0.08 & 0.06 & 0.08 & -0.04 & 0.18 & 0.06 & 0.06 & 0.06 \\
\hline III & 0.04 & 0.10 & 0.06 & 0.10 & 0.00 & 0.17 & 0.04 & 0.04 & 0.05 \\
\hline IV & 0.04 & 0.08 & 0.06 & 0.08 & 0.06 & 0.16 & 0.04 & 0.03 & 0.04 \\
\hline
\end{tabular}




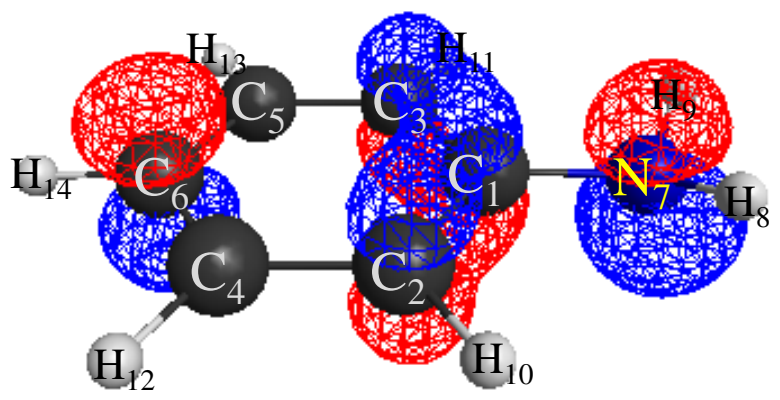

Figure 1: HOMO orbital of aniline. 
(a)

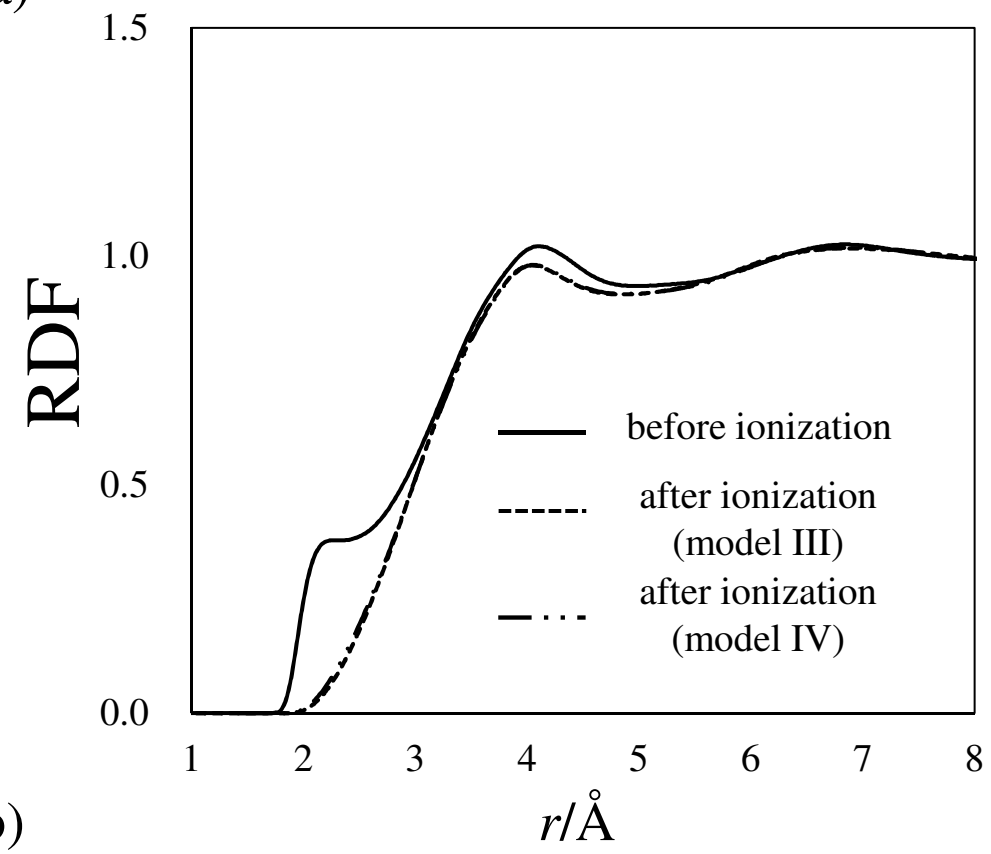

(b)

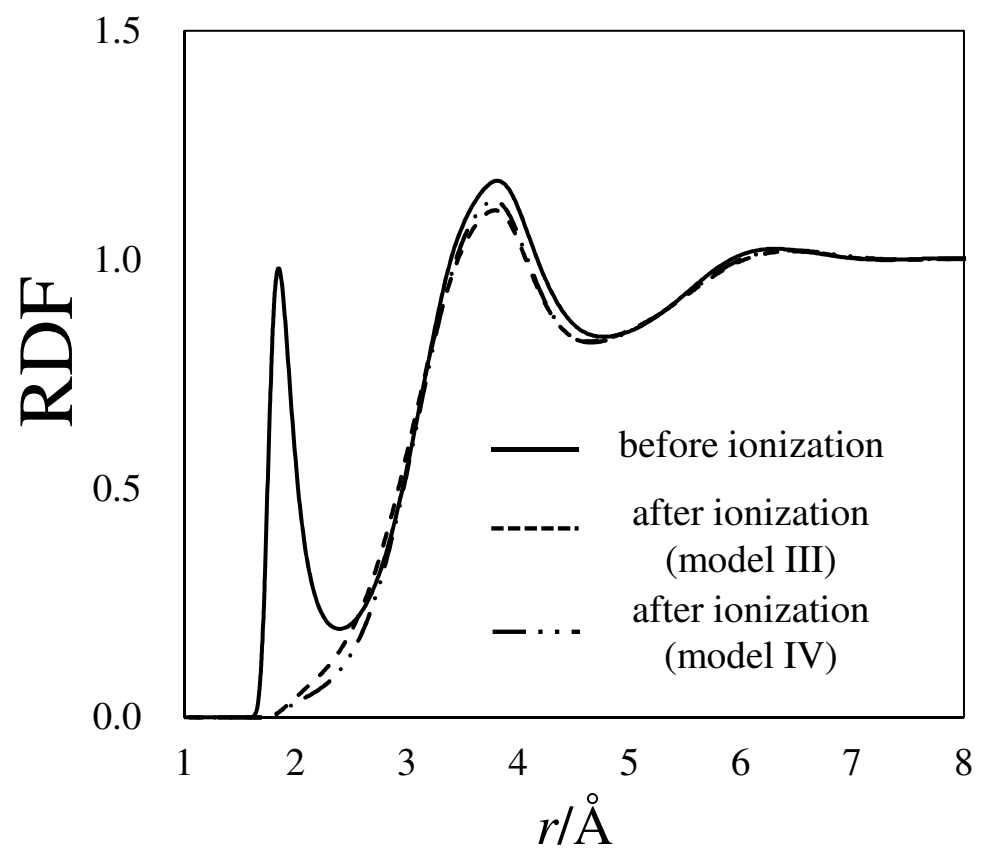

Figure 2: RDFs between (a) $\mathrm{C}_{6}$ and $\mathrm{H}_{\mathrm{w}}$, (b) $\mathrm{N}_{7}$ and $\mathrm{H}_{\mathrm{w}}$. 